Publications of the Astronomical Society of the Pacific, 117:1223-1229, 2005 November

(C) 2005. The Astronomical Society of the Pacific. All rights reserved. Printed in U.S.A.

\title{
Time-Resolved Spectroscopy and Photometry of CT Serpentis and V825 Herculis
}

\author{
F. A. Ringwald, Daniel W. Chase, and David S. Reynolds \\ Department of Physics, California State University, 2345 East San Ramon Avenue, MS MH37, Fresno, CA 93740-8031; ringwald@csufresno.edu \\ Received 2005 May 30; accepted 2005 July 29; published 2005 October 14
}

\begin{abstract}
Time-resolved spectroscopy and photometry of two cataclysmic variables (CVs) are presented. A radial velocity study shows CT Ser (Nova Ser 1948) has an orbital period of $0.195 \pm 0.002$ days. Its light curve shows only erratic flickering. A radial velocity study shows that V825 Her (PG 1717+413) has an orbital period of $0.206 \pm 0.002$ days. This CV's spectrum is similar to that of a dwarf nova in outburst, or an old nova: the long-term light curve shows only small, "stunted" outbursts, so it is likely to be a luminous nova-like CV, similar to a dwarf nova stuck in outburst all the time. We use time-resolved photometry to discover quasi-periodic oscillations (QPOs), with periods between 15 and 24 minutes, not unlike those seen in other luminous CVs.
\end{abstract}

\section{INTRODUCTION}

Many properties of cataclysmic variable binary stars (CVs) depend on their orbital periods $\left(P_{\text {orb }}\right)$, including their secular evolution, luminosity, and outbursts (Shafter et al. 1986). The catalogs of Patterson (1984) and Ritter \& Kolb (2003) include only CVs of known or suspected period, emphasizing this parameter's importance.

Radial velocity studies reveal orbital periods more reliably than do photometric modulations, save for eclipses, but are not without problems. Orbital semiamplitudes, or $K$-velocities, do not reliably trace the motion of the white dwarf, since they originate in the disk. The same problem applies to $\gamma_{\mathrm{em}}$, the emission-line mean velocity, and to $T_{0}$, the epoch of spectroscopic phase zero. Eclipsing systems sometimes show $T_{0}$ lagging the eclipse by over $70^{\circ}$ in phase (e.g., Thorstensen et al. 1991). Reviews on CVs that discuss these issues include those by Warner (1995) and Hellier (2001).

This paper reports spectroscopic period measurements of two CVs, CT Serpentis (Nova Ser 1948) and V825 Herculis (PG $1717+413)$. Time-resolved, differential photometry shows quasi-periodic oscillations (QPOs) in the light curve of V825 Her, but not in CT Ser. Table 1 presents the derived orbital parameters for both stars, and Table 2 lists the radial velocities measured for them. In $\S 2$, observational procedure, data reduction, and analysis are described. The individual CVs are discussed in $\S \S 3-4$, with conclusions presented in $\S 5$.

\section{OBSERVATIONS}

\subsection{Spectra}

The spectroscopic observations were carried out at Michigan-Dartmouth-MIT Observatory with the $1.3 \mathrm{~m} \mathrm{McGraw-Hill}$ telescope and the Mark IIIa spectrograph. The long-slit spectra normally permit accurate sky subtraction, although absolute spectrophotometry can be trusted only to $30 \%$, because of slit losses through the 2".2 slit used for all exposures. The spectrograph was never rotated to match the parallactic angle; it was deemed unnecessary for this setup, because our spectral coverage was so red. The detector was a TI-4849 CCD in the BRICC camera (Luppino 1989).

A 300 line $\mathrm{mm}^{-1}$ grism blazed at $6400 \AA$ was in the spectrograph. A Hoya Y-50 order-sorting filter was also used, which is important since CVs have very blue continua. The wavelength coverage spanned about $6200-8900 \AA$. The wavelength resolution was $11 \AA$, at a $5 \AA$ per channel dispersion. Exposure times were $900 \mathrm{~s}$ for all spectra.

The spectra of CT Ser were taken on 1991 May 22-25, and the spectra of V825 Her were taken on 1991 May 28-30. All spectra were reduced and analyzed as in Thorstensen \& Freed (1985) and Ringwald et al. (1994). For all CVs, velocities were measured by convolving the $\mathrm{H} \alpha$ emission line with two Gaussians that were 2.8 channels $\left(650 \mathrm{~km} \mathrm{~s}^{-1}\right)$ wide, separated by amounts given in Tables 1-2. All times are given as the heliocentric time of midexposure. Calibration spectra from a neon lamp were taken between each pair of spectra taken, or approximately every half hour, on all nights. The wavelength calibrations were done by fits to fifth-order polynomials, using the average shift of 10 lines to the blue and 23 lines to the red of the $\mathrm{H} \alpha$ line, and the rms residuals to these fits were always less than $10 \mathrm{~km} \mathrm{~s}^{-1}$, which matched the shifts shown by measurements of the night-sky line at $5577 \AA$. Errors for the individual velocities were found by fitting Gaussians to the line profiles and finding the difference between this and the line profiles measured by the double-Gaussian deconvolution: the small variations in the center of the line, probably due to turbulent motions in the accretion disk, as well as count statistics of the line, typically give errors of less than $10 \mathrm{~km} \mathrm{~s}^{-1}$ (see Ringwald et al. 1994).

Not all spectroscopic observations were made in photometric conditions, but flux standards from Oke (1974) were taken at 
TABLE 1

Derived Orbital Parameters for H $\alpha$ Velocities

\begin{tabular}{cccccc}
\hline \hline Object & $\begin{array}{c}P_{\text {orb }} \\
(\text { days })\end{array}$ & $\begin{array}{c}K_{\text {em }} \\
\left(\mathrm{km} \mathrm{s}^{-1}\right)\end{array}$ & $\begin{array}{c}\gamma_{\text {em }} \\
\left(\mathrm{km} \mathrm{s}^{-1}\right)\end{array}$ & $\begin{array}{c}T_{0} \\
(\mathrm{HJD}-2,440,000)\end{array}$ & $\begin{array}{c}\Sigma \\
\left(\mathrm{km} \mathrm{s}^{-1}\right)\end{array}$ \\
\hline $\mathrm{CT} \mathrm{Ser}^{\mathrm{a}} \ldots \ldots \ldots$ & $0.195 \pm 0.002$ & $55 \pm 10$ & $2 \pm 7$ & $8400.790 \pm 0.006$ & 57 \\
V825 Her $^{\mathrm{b}} \ldots \ldots$ & $0.206 \pm 0.002$ & $67 \pm 10$ & $177 \pm 7$ & $8406.676 \pm 0.005$ & 45 \\
\hline \hline
\end{tabular}

Notes. - Velocities were fitted to $v(t)=\gamma_{\mathrm{em}}+K_{\mathrm{em}} \sin \left[2 \pi\left(t-T_{0}\right) / P_{\mathrm{orb}}\right]$. All measurements used the method of Schneider \& Young (1980), in which the emission line was convolved with two Gaussians, and the centroid of the convolution was taken to be the velocity of the line. The Gaussians had FWHMs of $640 \mathrm{~km} \mathrm{~s}^{-1}$. All errors were estimated to $68 \%$ confidence using the Monte Carlo analysis of Thorstensen \& Freed (1985).

${ }^{\text {a }}$ Gaussian separation of $650 \mathrm{~km} \mathrm{~s}^{-1}$.

${ }^{\mathrm{b}}$ Gaussian separation of $1500 \mathrm{~km} \mathrm{~s}^{-1}$.

TABLE 2

H $\alpha$ Emission Radial Velocities

\begin{tabular}{|c|c|c|c|c|c|c|c|}
\hline $\mathrm{HJD}^{\mathrm{a}}$ & $\begin{array}{c}v \\
\left(\mathrm{~km} \mathrm{~s}^{-1}\right)\end{array}$ & $\mathrm{HJD}^{\mathrm{a}}$ & $\begin{array}{c}v \\
\left(\mathrm{~km} \mathrm{~s}^{-1}\right)\end{array}$ & $\mathrm{HJD}^{\mathrm{a}}$ & $\begin{array}{c}v \\
\left(\mathrm{~km} \mathrm{~s}^{-1}\right)\end{array}$ & $\mathrm{HJD}^{\mathrm{a}}$ & $\begin{array}{c}v \\
\left(\mathrm{~km} \mathrm{~s}^{-1}\right)\end{array}$ \\
\hline \multicolumn{8}{|c|}{ CT Ser $=$ PG $1543+145=$ Nova Ser $1948(1991 \text { May } 22-25)^{\mathrm{b}}$} \\
\hline 8399.669 & 138 & 8399.859 & 131 & $8400.766 \ldots \ldots$ & -13 & 8400.945 & -79 \\
\hline 8399.680 & 11 & $8399.870 \ldots \ldots$ & 83 & $8400.777 \ldots \ldots$ & -17 & 8400.956 & -70 \\
\hline $8399.692 \ldots \ldots$ & 71 & $8399.881 \ldots \ldots$ & 134 & $8400.789 \ldots \ldots$ & 3 & $8400.968 \ldots \ldots$ & -30 \\
\hline $8399.714 \ldots \ldots$ & -6 & $8399.892 \ldots \ldots$ & 39 & $8400.799 \ldots \ldots$ & -4 & 8401.699 & -134 \\
\hline 8399.724 & -8 & $8399.903 \ldots \ldots$ & 72 & $8400.811 \ldots \ldots$ & 39 & $8401.710 \ldots \ldots$ & -155 \\
\hline 8399.736 & 72 & $8399.914 \ldots \ldots$ & 27 & $8400.822 \ldots \ldots$ & 50 & 8401.722 & -44 \\
\hline 8399.747 & 21 & $8399.925 \ldots \ldots$ & -39 & $8400.833 \ldots \ldots$ & -6 & 8401.732 & -65 \\
\hline 8399.758 & -38 & $8399.936 \ldots \ldots$ & 56 & $8400.844 \ldots \ldots$ & 7 & 8401.744 & -56 \\
\hline $8399.769 \ldots \ldots$ & -97 & $8399.947 \ldots \ldots$ & -41 & $8400.856 \ldots \ldots$ & 122 & 8401.755 & -40 \\
\hline $8399.781 \ldots \ldots$ & -89 & $8399.958 \ldots \ldots$ & 24 & $8400.866 \ldots \ldots$ & 104 & 8401.767 & -14 \\
\hline $8399.791 \ldots \ldots$ & -12 & $8399.969 \ldots \ldots$ & 101 & $8400.878 \ldots \ldots$ & -29 & 8401.777 & 19 \\
\hline 8399.803 & -48 & $8399.979 \ldots \ldots$ & 44 & $8400.889 \ldots \ldots$ & -60 & 8401.790 & -27 \\
\hline 8399.814 & 92 & $8400.721 \ldots \ldots$ & -59 & $8400.901 \ldots \ldots$ & -80 & 8401.800 & 2 \\
\hline 8399.825 & 60 & $8400.732 \ldots \ldots$ & -57 & $8400.912 \ldots$ & -70 & 8401.812 & -20 \\
\hline $8399.836 \ldots \ldots$ & 13 & $8400.744 \ldots \ldots$ & -125 & $8400.923 \ldots \ldots$ & -65 & 8401.823 & -54 \\
\hline $8399.848 \ldots \ldots$ & 102 & $8400.755 \ldots \ldots$ & -10 & $8400.934 \ldots \ldots$ & -96 & & \\
\hline \multicolumn{8}{|c|}{ V825 Her $=$ PG $1717+413(1991 \text { May } 28-30)^{\mathrm{c}}$} \\
\hline 8405.769 & 155 & 8405.891 & 193 & 8406.739 & 281 & 8406.859 & 151 \\
\hline 8405.780 & 120 & $8405.902 \ldots \ldots$ & 166 & 8406.752 & 256 & 8406.872 & 131 \\
\hline $8405.792 \ldots \ldots$ & 122 & $8405.915 \ldots \ldots$ & 189 & $8406.763 \ldots \ldots$ & 265 & 8406.884 & 129 \\
\hline $8405.804 \ldots \ldots$ & 112 & $8405.926 \ldots \ldots$ & 207 & $8406.776 \ldots \ldots$ & 193 & 8406.896 & 150 \\
\hline $8405.816 \ldots \ldots$ & 119 & $8405.959 \ldots \ldots$ & 144 & $8406.787 \ldots \ldots$ & 183 & $8406.908 \ldots \ldots$ & 157 \\
\hline $8405.827 \ldots \ldots$ & 122 & $8406.680 \ldots \ldots$ & 195 & $8406.800 \ldots \ldots$ & 165 & 8406.919 & 196 \\
\hline $8405.839 \ldots \ldots$ & 102 & $8406.691 \ldots \ldots$ & 245 & $8406.811 \ldots \ldots$ & 122 & 8406.944 & 250 \\
\hline $8405.851 \ldots \ldots$ & 153 & $8406.703 \ldots \ldots$ & 265 & $8406.824 \ldots \ldots$ & 108 & 8406.956 & 195 \\
\hline $8405.864 \ldots \ldots$ & 157 & $8406.715 \ldots \ldots$ & 256 & $8406.835 \ldots \ldots$ & 124 & & \\
\hline $8405.875 \ldots \ldots$ & 199 & $8406.727 \ldots \ldots$ & 251 & $8406.847 \ldots \ldots$ & 123 & & \\
\hline
\end{tabular}

${ }^{\text {a }}$ Heliocentric Julian Date of mid-integration, minus 2,440,000.

${ }^{\mathrm{b}}$ Double Gaussian algorithm, with separation of $650 \mathrm{~km} \mathrm{~s}^{-1}$.

${ }^{c}$ Double Gaussian algorithm, with separation of $1500 \mathrm{~km} \mathrm{~s}^{-1}$. 


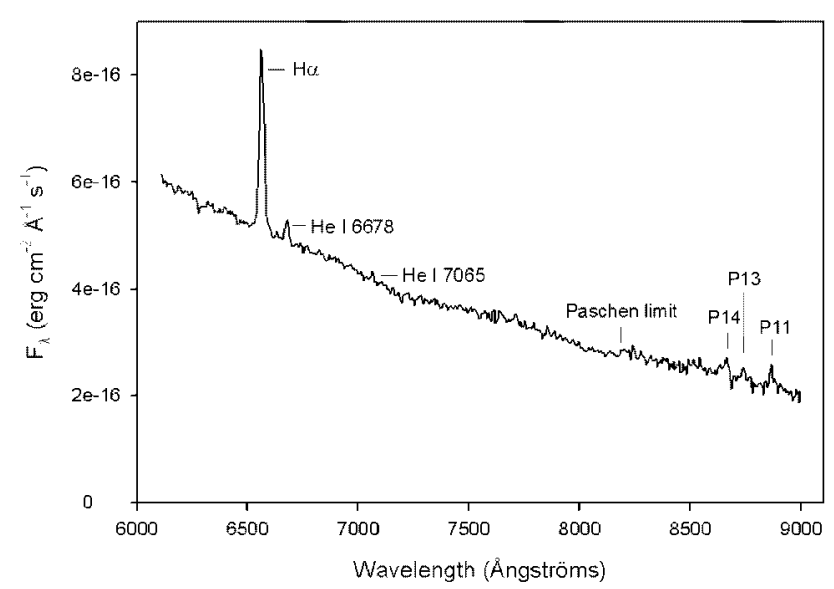

FIG. 1.-Average of the 63 spectra of CT Ser, comprising $15.75 \mathrm{hr}$ exposure time. A hot stellar spectrum was used to map and remove the telluric absorption, with some residual absorption near 6870 and $7600 \AA$. No lines from the mass-losing secondary star are obvious, not unusual for an old nova, which often have high accretion luminosities.

least once per night, to calibrate the instrumental response. Red spectra of hot stars, such as sdO stars from Stone (1977) or metal-deficient sdF stars from Oke \& Gunn (1983), were also taken. These were used to map and remove atmospheric absorption bands (see Wade \& Horne 1988).

\subsection{Photometry}

The time-resolved photometry was obtained with the CCD 2001 imaging detector on the 40 inch $(1 \mathrm{~m})$ telescope at Mount Laguna Observatory. No filter was used, for the highest throughput possible, with the maximum sensitivity near 6000 $7000 \AA$. The photometry of CT Ser was obtained on 2003 June 13-15 UT. The photometry of V825 Her was obtained on 2003 June 15-17 UT. The same square subarray of the CCD, 600 pixels on a side, was used for all observations on these four nights. This shortened the dead time between exposures while reading out the $\mathrm{CCD}$. The exposure times were chosen as a compromise between signal-to-noise ratio $(\mathrm{S} / \mathrm{N})$ and time resolution. The time resolution was $20 \mathrm{~s}$ for CT Ser (with $12 \mathrm{~s}$ exposures and $8 \mathrm{~s}$ of dead time between exposures to read out the $\mathrm{CCD}$ ) and $30 \mathrm{~s}$ for V825 Her (22 s exposures and $8 \mathrm{~s}$ of dead time between exposures). The weather was photometric, with no clouds from dusk to dawn, on all four nights. The seeing ranged between 1".8 and 2".4, which was above average for this setup.

All data were reduced using AIP4WIN version 1.4.23 software (Berry \& Burnell 2000). Basic calibrations involving simple dark subtraction and no flat fields were sufficient to measure $\mathcal{V}-\mathcal{C}$ magnitudes within $2.0 \%$ accuracy, found from scatter in the $\mathcal{V}-\mathcal{C}$ magnitudes. All frames were prechecked to ensure that the $\mathcal{V}, \mathcal{C}$, and $\mathcal{K}$ stars (variable, comparison, and check,

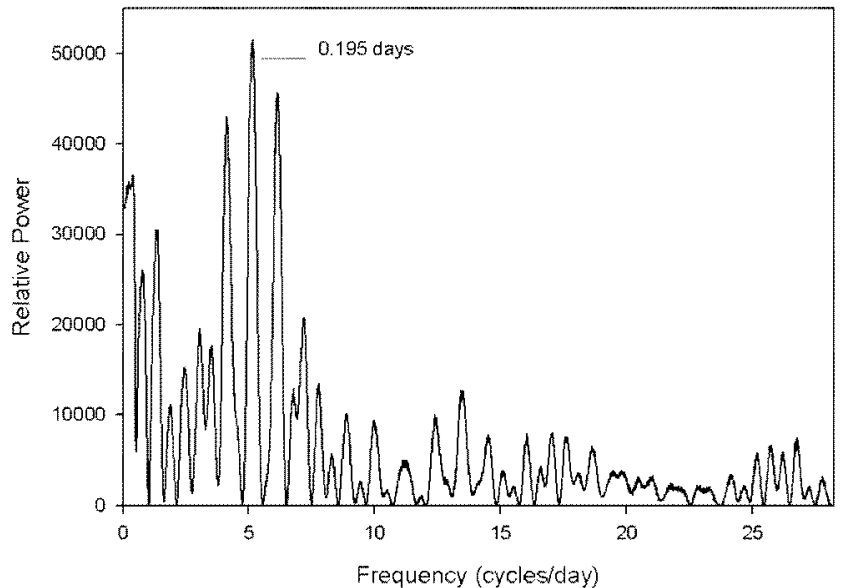

FIG. 2.-Lomb-Scargle periodogram for the $\mathrm{H} \alpha$ velocities of CT Ser. The highest peak, indicating the likely orbital period, corresponds to a period of 0.195 days.

respectively) remained fully visible and to discard exposures with contaminating cosmic rays. Any images with obtrusive hot/dark pixel regions were also discarded. Lomb-Scargle periodograms (Lomb 1976; Scargle 1982; Press \& Teukolsky 1988) were used to search for sinusoidal signals in the light curves of each object.

\section{CT SERPENTIS (NOVA SER 1948, PG 1543+145)}

CT Ser is the only classical nova listed in the atlas of Bode et al. (1989) that also appeared in the Palomar-Green catalog (Green et al. 1986). CT Ser had a nova eruption in 1948 and

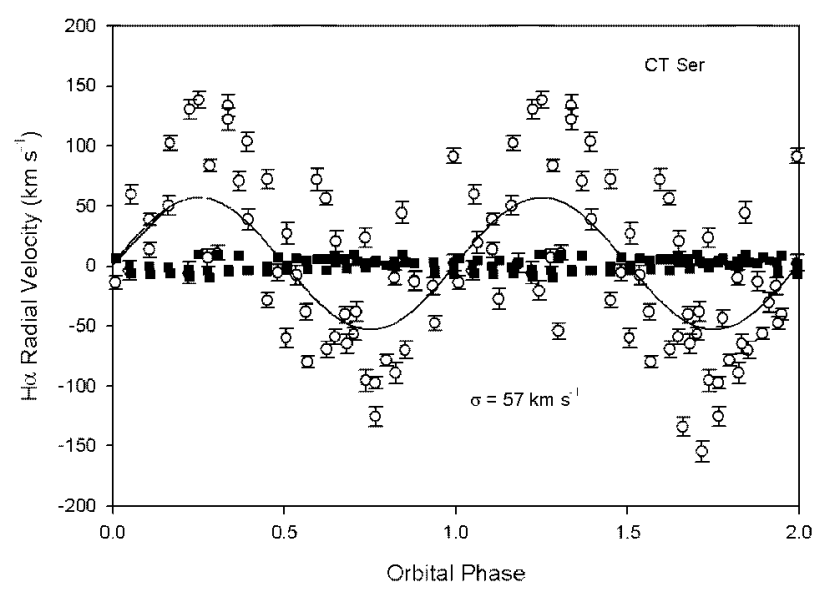

FIG. 3.-Least-squares fit to a sinusoid by the $\mathrm{H} \alpha$ velocities of CT Ser. All velocities are plotted twice for continuity using open circles, with error bars estimated by Gaussian fits. Also plotted with filled squares are velocities measured from the night-sky line at $5577 \AA$. 

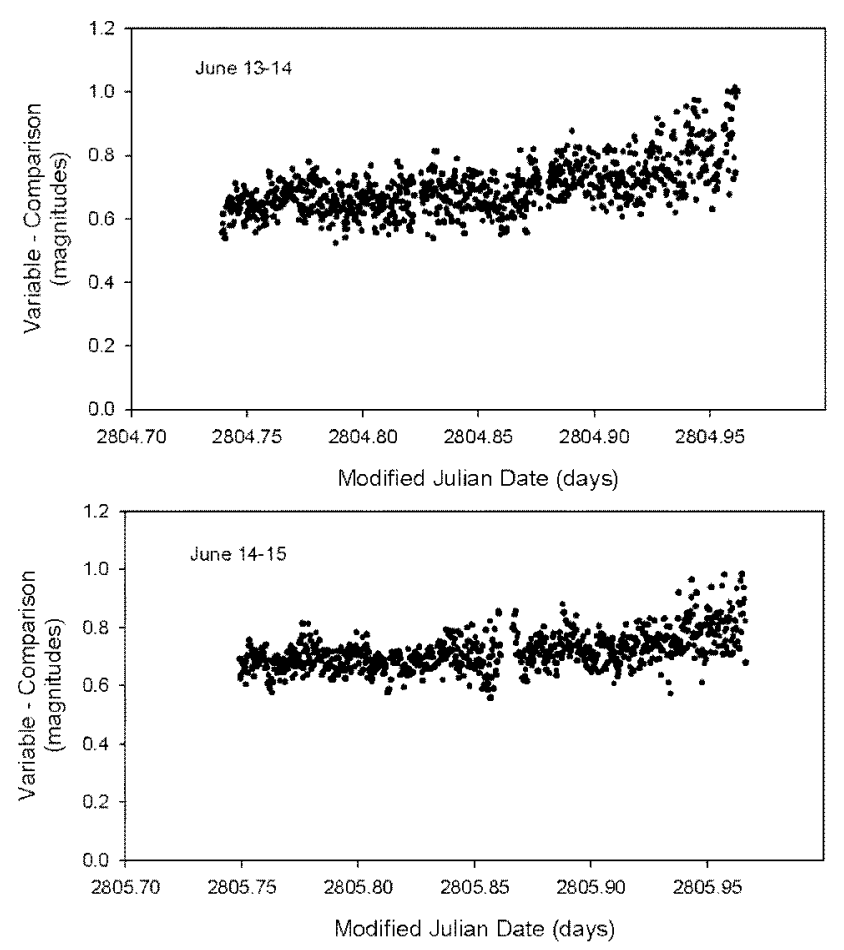

FIG. 4.-Light curve for CT Ser on 2003 June 13-14 UT (top) and June 14-15 UT (bottom). Both light curves show only erratic flickering, ubiquitous in CVs, and a rise throughout each night, probably from unequal atmospheric extinction in the target and comparison stars (but then, it is not easy to find a comparison star as blue as a typical CV). Errors in magnitude $(\mathcal{C}-\mathcal{K})$ are $\pm 0.02 \mathrm{mag}$.

was still relatively bright $\left(B_{\mathrm{pg}}=15.9\right)$ in $1973-1974$, when the Palomar Green Survey photographs were taken. Since 1988, CT Ser has been fainter than $V=16.0$ (VSNET). ${ }^{1}$ Even so, a spectrum taken in 1990 showed that it still had He II $\lambda 4686$ in emission, comparable in equivalent width to $\mathrm{H} \beta$ at about $5 \AA$ (Ringwald et al. 1996). In other words, although it was faint, there was still something quite hot in the system. It was at $V=16.4$ in this spectrum and was between $V=16.0$ and 16.5 at the time of our spectroscopic observations in 1990-1991, judging from the flux level of the spectra. Since the comparison stars of Henden \& Honeycutt (1997) were in our field of view, we estimate that CT Ser had $V \approx 16.8$ during our 2003 photometric observations, with $\mathcal{V}-\mathcal{C} \approx 0.65$ at the baseline of our curve.

The average spectrum of those used in the radial velocity study is shown in Figure 1. The spectrum does not differ significantly with the one for CT Ser given by Ringwald et al. (1996), being typical for an old nova, with Balmer and He I

\footnotetext{
${ }^{1}$ The Variable Star Network online database (Nogami et al. 2003) is available at http://www.kusastro.kyoto-u.ac.jp/vsnet/index.html.
}

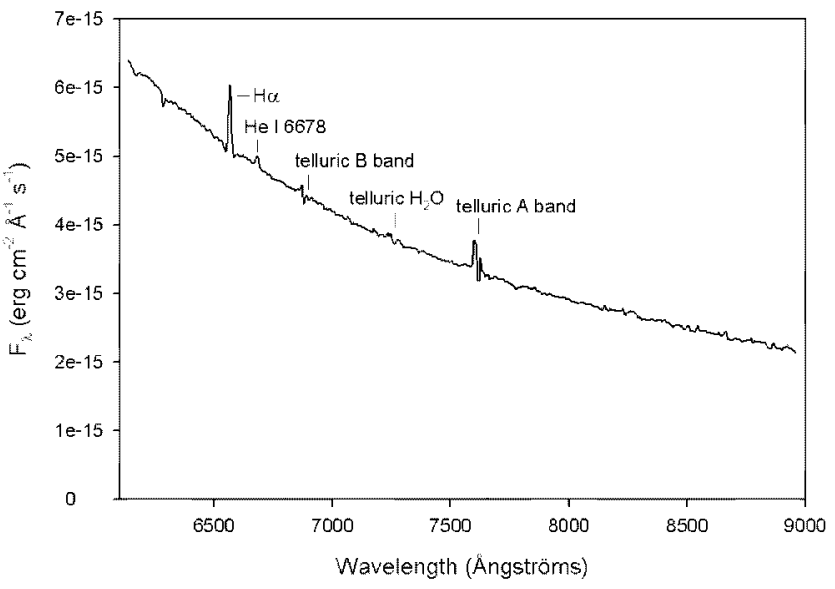

FIG. 5.-Average of the 38 spectra used in the radial velocity study of V825 Her, comprising $9.5 \mathrm{hr}$ exposure time. A hot stellar spectrum was used to map and remove the telluric absorption, with some residual absorption near 6870 and $7600 \AA$. This spectrum is similar to that of an optically thick CV accretion disk or a dwarf nova in outburst, with weak lines on a bright continuum and no obvious absorption features from a mass-losing secondary star, probably much fainter than the continuum.

emission lines on a bright, blue continuum, and no trace of the mass-losing secondary star.

A Lomb-Scargle periodogram (Press \& Teukolsky 1988) was computed to search for periodic signals in the radial velocities. This periodogram (see Fig. 2) shows a clear preferred peak above its aliases, even though the fit of the velocities to a sinusoid is noisy, with $K_{\mathrm{em}} / \sigma=0.97$ (see Fig. 3). The $K_{\mathrm{em}}$ was small, at $55 \mathrm{~km} \mathrm{~s}^{-1}$. Spectrograph flexure was found to be negligible, within $\pm 10 \mathrm{~km} \mathrm{~s}^{-1}$, by measuring velocities by fitting Gaussians to the night-sky line at $5577 \AA$ (see Fig. 3). Our time-resolved photometry of CT Ser revealed only erratic flickering (see Fig. 4), not uncommon for a CV.

\section{V825 HERCULIS (PG 1717+413)}

This nova-like CV was one of two found by Ferguson et al. (1984) in their search for "thick disk" CVs, which with their high mass-transfer rates and accretion luminosities resemble dwarf novae stuck in outburst all the time. The average of all our spectra taken in the radial velocity study is shown in Figure 5. It shows a typical spectrum for a nova-like CV, or a dwarf nova in outburst, with relatively weak Balmer and $\mathrm{He}$ I emission lines on a bright, power-law continuum. This pattern is often seen in luminous accretion disks, with no sign of absorption lines from the mass-losing secondary star.

The interpretation of the Lomb-Scargle periodogram calculated from the radial velocities (Fig. 6) is straightforward, with the most likely peak clearly higher than its aliases. At $K_{\mathrm{em}} / \sigma=1.49$, a sinusoidal radial velocity curve is definitely present (see Fig. 7). 


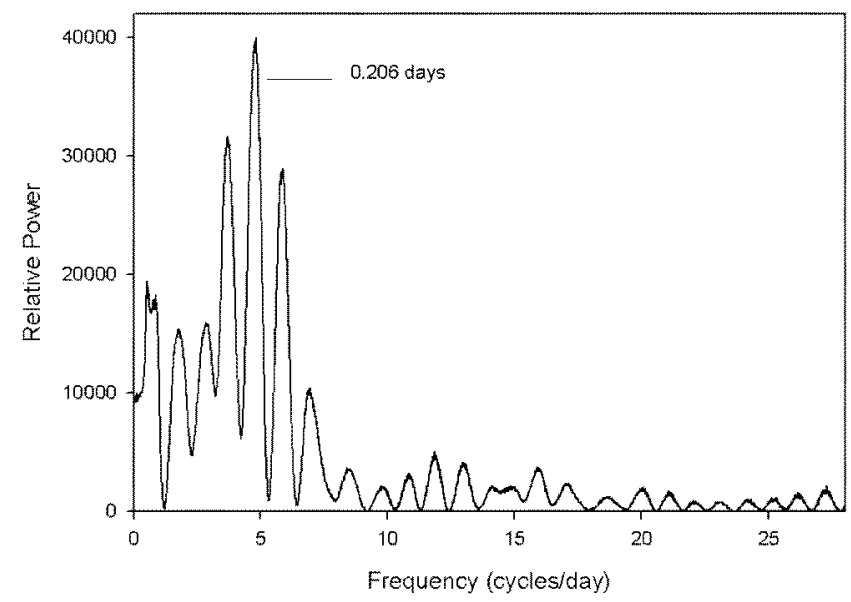

FIG. 6.-Lomb-Scargle periodogram for the $\mathrm{H} \alpha$ velocities of V825 Her. The highest peak, indicating the likely orbital period, corresponds to a period of 0.206 days.

Since the comparison stars of Henden \& Honeycutt (1997) were in our field of view, we estimate that V825 Her had $V \approx 16.4$ during our 2003 photometric observations (with $\mathcal{V}-\mathcal{C} \approx 1.6$ at the baseline). A periodogram of the time-resolved photometry shows power at frequencies below $7.89 \mathrm{cy}-$ cles day $^{-1}$, corresponding to periods greater than $3.0 \mathrm{hr}$ (see Fig. 8, top). This may be due to the orbit or the erratic flickering all CVs show in their light curves. There is no clear evidence of eclipses, superhumps, or other oscillations on the orbital timescale, but only two nights of photometry were taken: a longer campaign would be useful to search for any such effects.

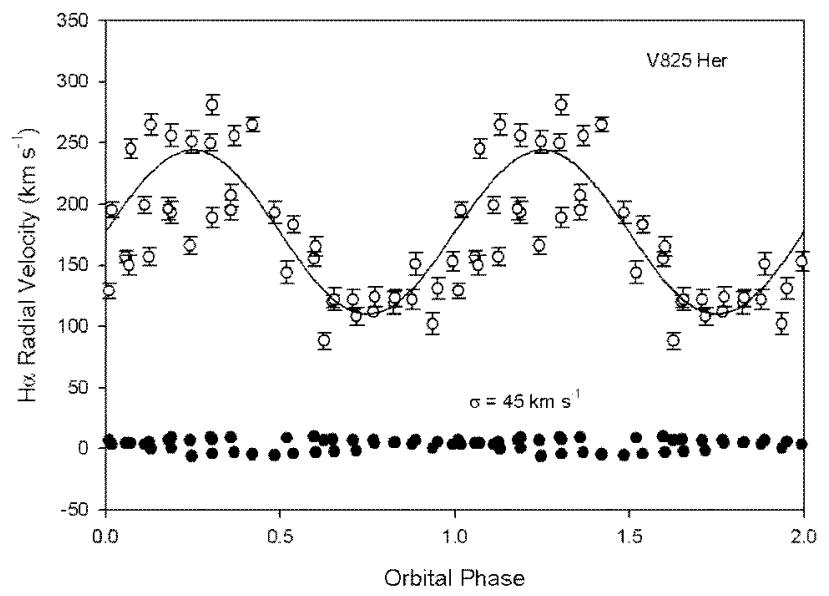

FIG. 7.-Least-squares fit to a sinusoid by the $\mathrm{H} \alpha$ velocities of V825 Her. All velocities are plotted twice for continuity using open circles, with error bars estimated by Gaussian fits. Also plotted with filled squares are velocities measured from the night-sky line at $5577 \AA$.

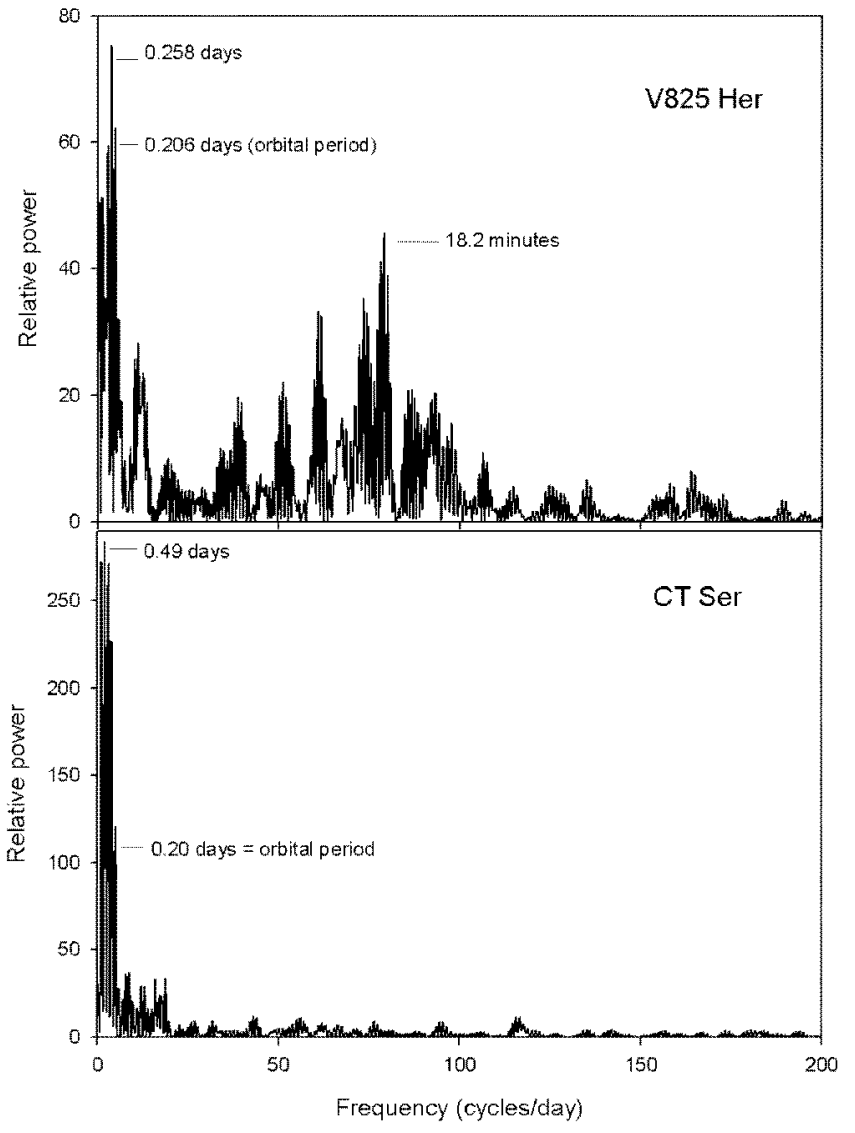

FIG. 8. - Lomb-Scargle periodograms for the time-resolved differential photometry of V825 Her (top) and CT Ser (bottom). V825 Her shows a family of periodicities between periods of 15-24 minutes. CT Ser does not show anything, other than power at low-frequency attributable to the orbit or erratic flickering.

This periodogram also shows power at high frequencies, corresponding to periods between 15 and 24 minutes. This power was probably not from spurious instrumental or atmospheric effects, since the periodogram of CT Ser, taken with the same instrumental setup and only two nights earlier, and under quite similar conditions, shows no obvious excess power at any frequency significantly higher than that of the orbital period (Fig. 8, bottom). The excess power at high frequencies in V825 Her was also present in periodograms for both nights separately (Fig. 9). Although the light curve of CT Ser shows only erratic flickering and a secular upturn that may well be atmospheric (see Fig. 4), oscillations are quite clearly discernible in the light curve of V825 Her (see Fig. 10).

Our 2003 photometric observations of V825 Her showed no sudden flaring reported as a sudden brightening of the $\mathrm{H} \alpha$ emission line by Ringwald (1991). Chen et al. (1993) noted that the light curve of V825 Her is variable on timescales of both minutes and days, and that the amplitude of the rapid 


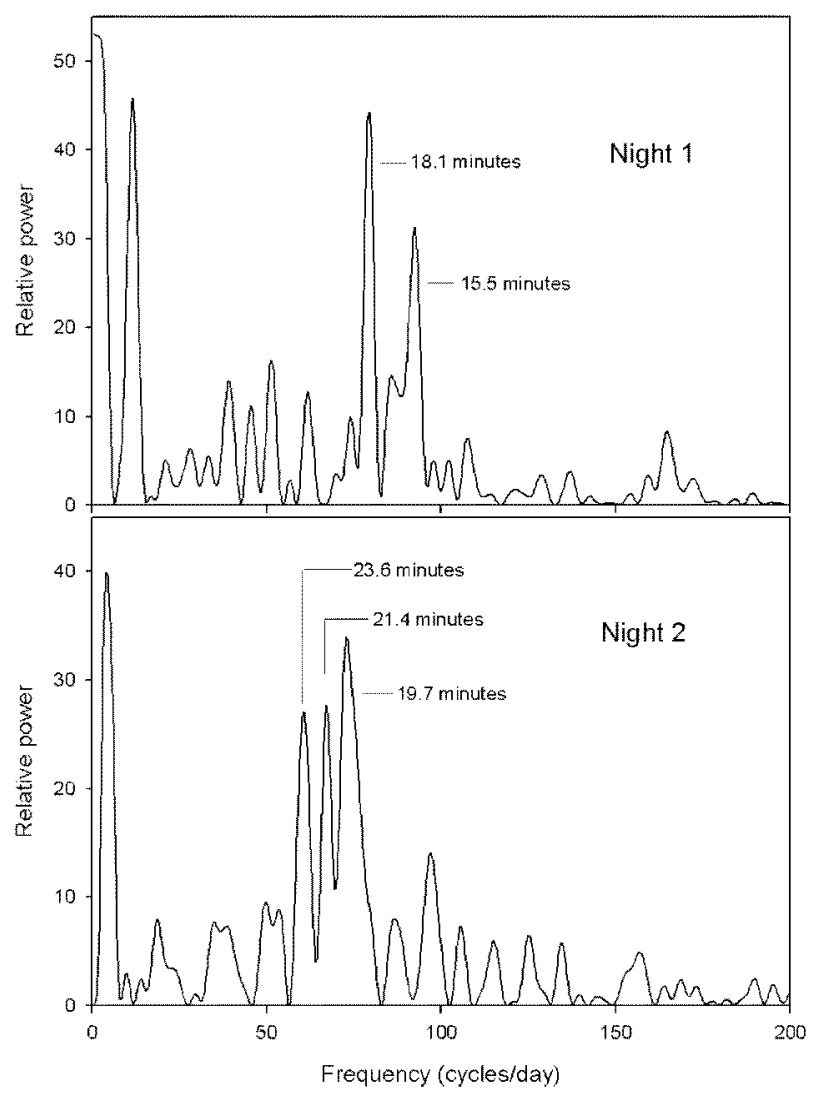

FIG. 9.-Lomb-Scargle periodograms for the time-resolved differential photometry of V825 Her on 2003 June 15-16 UT (top) and 2003 June 16-17 UT (bottom).

variability can change significantly over a 2 month timescale. Chen et al. also found no correlation with the apparent magnitude of the system. Honeycutt (2001) discovered "stunted" outbursts similar to those seen in other nova-like CVs.

\section{CONCLUSIONS}

This paper presents definitive orbital periods of V825 Her and CT Ser, measured with radial velocity studies, and reports on searches for photometric oscillations in both systems. These revealed oscillations with a period of 15-24 minutes in the light curve of V825 Her, but nothing other than erratic, lowfrequency noise for CT Ser. The 15-24 minute oscillations of V825 Her are clearly visible in the light curves (Fig. 10). They are probably not due to instrumental or atmospheric effects, since CT Ser was observed only 2 days earlier with the same equipment and under quite similar atmospheric conditions, and shows only erratic flickering, not unusual in an old nova.

The oscillations in V825 Her are most likely quasi-periodic oscillations (QPOs), the precise nature of which are unknown
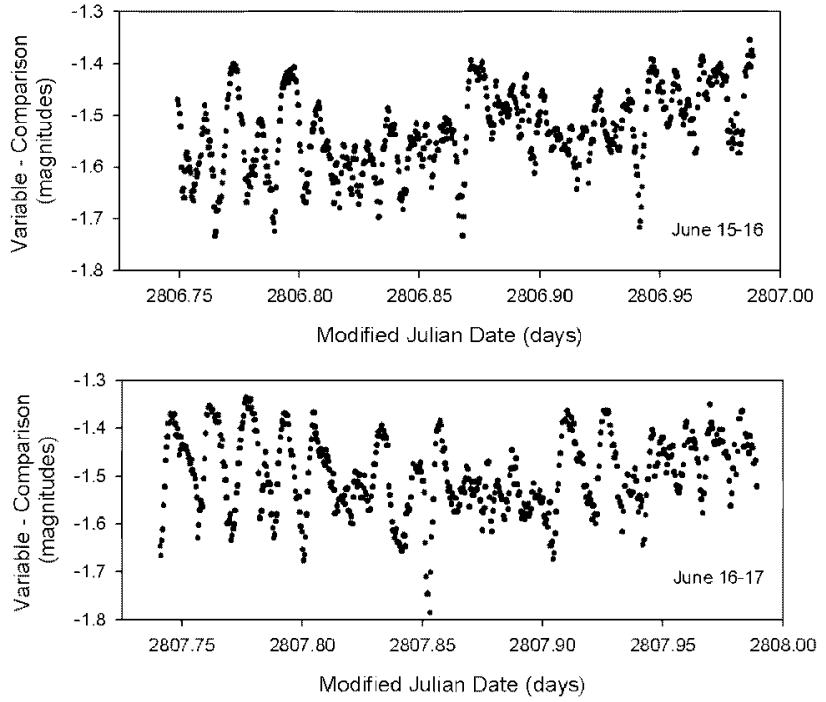

FIG. 10.- Light curve for V825 Her on 2003 June 15-16 UT (top) and June 16-17 UT (bottom). The 15-24 minute quasi-periodic oscillations are clearly present in both images. Errors in magnitude $(\mathcal{C}-\mathcal{K})$ are \pm 0.02 mag.

(Warner 1995; Hellier 2001). We estimate that $Q=f / \Delta f=17$, not unusual for QPOs, which typically have $Q \approx 1-10$. These QPOs have amplitudes of $0.1-0.3 \mathrm{mag}$, which is large for QPOs, but not unprecedented. Their range of periods is also not unusual for QPOs (Warner 1995, p. 437).

V825 Her is likely a nonmagnetic or weakly magnetic CV. Silber (1992) searched for coherent $\left(Q>10^{10}\right)$ optical pulsations from a magnetic white dwarf and found none. V825 Her is a HEAO A-1 X-ray source (Silber 1992), but its optical spectrum shows only a weak He II $\lambda 4686$ emission line, unlike those found in magnetic CVs. There is no trace of the mass-losing secondary star in our spectrum of V825 Her (see Fig. 5). The luminosity and mass transfer rate are therefore likely to be relatively high. Ferguson et al. (1984) found V825 Her to have a spectrum of a "thick-disk" CV similar to that of a dwarf nova in outburst, with faint emission cores in faint absorption wings in a bright continuum. It also shows "stunted" outbursts (Honeycutt 2001). V825 Her might therefore be a promising target for an extended, multilongitude photometric study, such as those by the Center for Backyard Astrophysics (Harvey et al. 1995), to observe whether its QPOs, apparent magnitude, and other variabilities are related.

The photometric observations were made at Mount Laguna Observatory, which is operated by the Department of Astronomy at San Diego State University. The Mount Laguna observations also used the High-Performance Wireless Research and Education Network (HP-WREN), funded by the National Science 
Foundation, under grant numbers 0087344 and 0426879 . We thank Danny Martino for her help on the observing run. The radial velocity observations were made at MDM Observatory,
Kitt Peak, Arizona, which is owned and operated by a consortium of the University of Michigan, Dartmouth College, Ohio State University, Columbia University, and Ohio University.

\section{REFERENCES}

Berry, R., \& Burnell, J. 2000, The Handbook of Astronomical Image Processing (Richmond: Willmann-Bell), chap. 8

Bode, M. F., Duerbeck, H. W., \& Evans, A. 1989, in Classical Novae, ed. M. F. Bode \& A. Evans (Chichester: Wiley), 249

Chen, J.-S., Jiang, Z.-J., Li ,Y., Liu, X.-W., \& Feng, X.-C. 1993, Acta Astrophys. Sinica, 13, 225

Ferguson, D. H., Green, R. F., \& Liebert, J. 1984, ApJ, 287, 320

Green, R. F., Schmidt, M., \& Liebert, J. 1986, ApJS, 61, 305

Harvey, D., Skillman, D. R., Patterson, J., \& Ringwald, F. A. 1995, PASP, 107, 551

Hellier, C. 2001, Cataclysmic Variable Stars: How and Why They Vary (Chichester: Springer), chap. 10

Henden, A. A., \& Honeycutt, R. K. 1997, PASP, 109, 441

Honeycutt, R. K. 2001, PASP, 113, 473

Lomb, N. R. 1976, Ap\&SS, 39, 447

Luppino, G. A. 1989, PASP, 101, 931

Oke, J. B. 1974, ApJS, 27, 21

Oke, J. B., \& Gunn, J. E. 1983, ApJ, 266, 713
Patterson, J. 1984, ApJS, 54, 443

Press, W. H., \& Teukolsky, S. A. 1988, Comput. in Phys., 2, 77

Ringwald, F. A. 1991, BAAS, 23, 1463

Ringwald, F. A., Naylor, T., \& Mukai, K. 1996, MNRAS, 281, 192

Ringwald, F. A., Thorstensen, J. R., \& Hamwey, R. M. 1994, MNRAS, 271,323

Ritter, H., \& Kolb, U. 2003, A\&A, 404, 301

Scargle, J. D. 1982, ApJ, 263, 835

Schneider, D. P., \& Young, P. J. 1980, ApJ, 238, 946

Shafter, A. W., Wheeler, J. C., \& Cannizzo, J. K. 1986, ApJ, 305, 261

Silber, A. D. 1992, Ph.D. thesis, Massachusetts Institute of Technology Stone, R. P. S. 1977, ApJ, 218, 767

Thorstensen, J. R., \& Freed, I. W. 1985, AJ, 90, 2082

Thorstensen, J. R., Ringwald, F. A., Wade, R. A., Schmidt, G. D., \& Norsworthy, J. E. 1991, AJ, 102, 272

Wade, R. A., \& Horne, K. 1988, ApJ, 324, 411

Warner, B. 1995, Cataclysmic Variable Stars (Cambridge: Cambridge Univ. Press) 